\title{
Teste MABC: aplicabilidade da lista de checagem na região sudeste do Brasil
}

\author{
Jane A.0. Silva1,2 \\ Luis E. Dantas 3,4 \\ Maria T. Cattuzzo 2,5 \\ Cinthya Walter ${ }^{2}$ \\ Cássia R.P. Moreira 6 \\ Cleverton J.F. Souza7
}

\author{
1 UNIVÁS - Universidade do Vale do Sapucaí \\ Pouso A legre, M inas Gerais, Brasil \\ 2 Laboratório de Comportamento M otor, EEFE-USP \\ São Paulo, Brasil \\ ${ }_{3} \mathrm{G}$ rupo de Estudos em D esenvolvimento da $\mathrm{A}$ ção M otora \\ e Intervenção - EEFE - USP, São Paulo, Brasil \\ ${ }^{4}$ Escola de Educação Física e Esportes, USP, São Paulo, Brasil \\ ${ }_{5}$ Escola Superior de E ducação Física \\ Universidade de Pernambuco, Recife, Brasil \\ 6 Universidade Paulista, UN IP - São Paulo, Brasil \\ 7 Universidade Estadual do A mazonas (UEA) \\ Campus de Tefé - A mazonas, Brasil
}

\begin{abstract}
RESUMO
Para algumas crianças ações típicas da infância são grandes desafios. Essa condição, com forte impacto no desenvolvimento infantil, extrapolando o domínio motor, foi classificada pela Associação Americana de Psiquiatria, Transtorno do Desenvolvimento da Coordenação (TDC). Estudos sobre sua natureza, diagnóstico e possibilidades de intervenção têm sido realizados. Um dos instrumentos diagnósticos mais utilizados é o Teste ABC do Movimento, composto por duas partes: lista de checagem e testes de habilidades motoras. A lista de checagem trata de aspectos comportamentais da criança e estudos têm sugerido que diferenças contextuais poderiam comprometer a sua aplicabilidade. Assim, o objetivo deste estudo foi investigar a aplicabilidade dessa lista por professores de uma região do sudeste do Brasil. Os sujeitos foram 30 professores de sala de aula os quais observaram três crianças de oito a nove anos, por sala. Três professores de Educação Física complementavam as observações, quando era necessário. Análise de conteúdo das listas apontou al gumas dificuldades na sua aplicabilidade pelos professores de sala, associadas a fatores como a formação/ expe riência profissional e infra-estrutura escolar. Os resultados mostraram que, apesar das dificuldades, os professores de sala reconhecem os comportamentos descritos e que a atuação conjunta do professor de Educação Física com o general ista mostrou ser a solução mais adequada para o preenchimento da lista de checagem.
\end{abstract}

Palavras-chave: transtorno do desenvolvimento coordenativo; teste $A B C$ do M ovimento; lista de checagem.

\section{ABSTRACT \\ MA BC test: check-list applicability in the southeast region of Brazil}

To some children typical actions of the childhood are big challenges. This condition, that has a strong impact in the child development overtaking the motor domain, was classified by the A merican Psychiatric A ssociation as motor coordination disorder (DCD). Studies about its origin, diagnostic and possibilities of intervention have been done. 0 ne of the most used diagnostic instruments has been the M ovement A ssessment Battery for Children, which is composed by two parts: checklist and tests of motor skills. The checklist broaches the behavioral aspects of the children and researches have been suggested that contextual differences could affect the applicability of the list. Thus, the aim of the present study was to investigate the applicability of the checklist by teachers of a region at the southwest of Brazil. The subjects were 30 classroom teachers that observed three children from eight to nine years old, per classroom. Physical Education teachers completed these observations, when it was necessary. The analysis of the content of the lists showed that the classroom teachers found some difficulties on the applicability of the checklist; which is related to professional formation and the structure of the school. The results showed that, in spite of some difficulties, the classroom teacher recognize the behaviors described and that the join action of the Physical Education teacher and the generalist, was the most adequate solution for the filling of the checking list.

Key-words: children; motor coordination disorder; M A BC test; checklist. 


\section{INTRODUÇÃO}

As habilidades motoras fundamentais são consideradas os blocos críticos na construção de habilidades mais avançadas. Portanto, é importante que sua prática seja oportunizada desde a primeira infância (27). Em geral pouca atenção é dada aos movimentos cotidianos, porém eles são imprescindíveis para uma ação eficiente no ambiente. Entretanto, para algumas crianças essas ações não são tão simples e fáceis, de modo que manusear talheres, escrever, receber uma bola e outras tarefas típicas da primeira e segunda infância se constituem grandes desafios. Essas dificuldades causam um forte impacto no desenvolvimento do indivíduo, que extrapolam o âmbito motor e influenciam o desempenho acadêmico, o desenvolvimento social e psicológico.

Crianças que apresentam atrasos motores tendem a evitar qualquer experiência que demande uma coordenação motora ótima. Entretanto, esta estratégia esbarra no fato de que o cotidiano humano, como já foi anteriormente destacado, demanda muitas ações motoras e, sob a perspectiva de uma criança, de uma crescente complexidade. A repercussão dessa dificuldade em executar ações motoras vai além da nítida dificuldade em interagir com ambiente físico e social presente, e abarca também uma grande probabilidade de dificuldades no seu desenvolvimento futuro (26). Portanto, investigar crianças que demonstram atrasos no desenvolvimento das habilidades motoras surge como resposta aos resultados de diversas pesquisas $(26,27,29,30,4,10,13,8,14)$ que sugerem que essas crianças são mais suscetíveis às pressões de contextos de aprendizagem, levando ao abandono e/ ou à não participação em atividades físicas.

Muitos termos têm sido usados para nomear esse problema, e esses termos refletem à área de estudo específica dos autores ou dos diferentes grupos de pesquisa. Os da área médica usam o termo desajeitado (clumsy), (4, 10, 13, 8, 9, 14, 2, 3, 6, 19) ao passo que os profissionais da área de educação, além desse termo, usam inábil (awkward) (28,12). Há ainda outros termos como, por exemplo, síndrome da criança desajeitada (clumsy child syndrome) (7) e criança com problemas ou dificuldades na coordenação (coordination problems or difficulties) (9,16). Somente na década de 1980 este fenômeno foi reconhecido pela Organização Mundial de Saúde - OMS e pela Associação de Psiquiatria
Americana - APA, e recebeu o nome de "Transtorno do Desenvolvimento da Coordenação" - TDC (1). A evidência de que a criança diagnosticada com TDC pode ter, além da desordem motora, dificuldades comportamentais, sociais e afetivas está registrada na literatura. $O$ estudo de Wright (30) mostrou que professores notaram diferenças comportamentais entre crianças com TDC, as quais apresentavam dificuldades motoras diante da rotina escolar, comparativamente a outras crianças que mostravam boa coordenação para enfrentar desafios dessa rotina. Kal verboer, De Vries e Van Dellen (11) relataram que crianças consideradas com TDC eram solitárias, submissas e auto-conscientes. Eles afirmaram, ainda, que elas necessitavam de autoconfiança para lidar com as dificuldades que encontravam diante das habilidades sociais, evitando o seu envolvimento com os jogos, as brincadeiras e as atividades esportivas. Essas crianças tinham a tendência de se isolar. Embora muitas pesquisas tenham sido realizadas desde a década de 1990 (5,11,16,17,20,21,22,23,24,25,29), a natureza do TDC ainda não está totalmente descrita. Pode-se destacar que as primeiras explicações de sua possível causa estão relacionadas a fatores pré-natais e à imaturidade cerebral (7).

Um ponto importante é que diante das várias conse qüências as quais a criança diagnosticada com TDC está sujeita, mesmo que as investigações mostrem limitações nos instrumentos e/ ou diferenças metodológicas, todas deveriam suscitar meios capazes, se não de isentar dos problemas adicionais, ao menos de ajudar essas crianças a lidar com suas dificuldades nos ambientes em que vivem, especial mente na escola. Alguns testes têm sido usados para avaliar o TDC em crianças. Dentre estes, um dos mais populares no estudo de crianças com TDC é a Bateria de Avaliação de Movimento para Crianças (M ovement A ssessement Battery for Children - M-ABC) (20). Esta Bateria de avaliação de Movimento para Crianças (M-ABC) criado por Henderson e Sugden em 1992 (9), consiste na verdade em dois testes distintos e complementares. Um é constituído de uma bateria de testes motores (M-ABC Teste) e o outro é um questionário na forma de uma lista de checagem ( $M$ ABC Checklist). A utilização de um teste como uma lista de checagem tem sido visto como um instrumento fundamental para detecção, o mais precoce 
possível, das crianças portadores de TDC. A necessidade de teste que possa ser aplicado a grandes populações é uma importante ferramenta, pois permite melhorar drasticamente a eficiência de políticas de intervenção precoce. Um dos contextos mais promissores para a identificação de crianças portadoras de TDC é a escola. O cotidiano escolar demanda praticamente todo o espectro de habilidades motoras, com a vantagem de abrigar um observador privilegiado, o professor. A lista de checagem do M-ABC foi criada pelos autores especificamente para ser utilizada no contexto escolar. Schoemaker et al. (20) investigaram a eficiência da lista de checagem do M$A B C$ e concluíram que o referido teste é recomendado para mapear as crianças com dificuldades motoras na escola. Entretanto, ressaltam:

"Para uma identificação apropriada das crianças com TDC, 0 mapeamento com a lista de checagem não é suficiente; $M$ A BC Teste precisa ser usado para confirmar o diagnóstico" (20) p.439.

A lista de checagem trata de aspectos comportamentais da criança e deve ser aplicada por uma pessoa adulta que conheça e acompanhe sua rotina, como, por exemplo, o professor de sala de aula e/ ou professor de Educação Física. Ela está dividida em cinco seções e cada uma delas contém doze itens.

A primeira seção contém itens que permitem caracterizar a criança parada em um ambiente estável. A segunda seção consta da avaliação da criança em movimento em um ambiente estável. A terceira seção aval ia a criança parada no ambiente em movimento. A quarta seção avalia a criança e o ambiente em movimento. Nessas quatro seções citadas, a pontuação é: muito bem $=0$; consegue $=1$; quase consegue $=2$; não consegue $=3$. A quinta seção trata de problemas de comportamento relacionados a dificuldades sociais e afetivas. Nessa seção, a pontuação é: raramente $=0$; ocasional mente $=1$; frequentemente $=2$. A outra parte do teste, que é uma bateria de habilida des motoras, está organizada em quatro seções de acordo com a faixa etária: a seção 1 está voltada para crianças de quatro a seis anos de idade; a seção 2 refere-se a crianças de sete e oito anos de idade; a seção 3 está relacionada a crianças de nove e dez anos de idade; e a seção 4 diz respeito a crianças de onze e doze anos de idade. Para cada seção há um conjunto de oito tarefas que envolvem habilidades manuais, habilidades com bola e equilíbrio estático e dinâmico. De acordo com o rendimento da criança é atribuído um valor como, por exemplo, números de tentativas, número de acertos, número de erros cometidos ou tempo gasto para executar a tarefa que, em seguida, é convertido para a escala de escores própria do teste, contabilizando o desempenho geral da criança. No Brasil, da crescente preocupação com as crianças com transtornos motores, têm surgido al guns estudos que se utilizaram do Teste $A B C$ do Movimento $(5,17,25)$. O objectivo deste estudo pretendeu verificar a aplicabilidade da Lista de Checagem do teste ABC do Movimento (9) em duas comunidades situadas no estado de Minas Gerais, na região Sudeste do Brasil. Pretendeu igualmente saber se o profissional que acompanha a rotina da criança está apto a responder à mesma e se ele dispõe de tempo para observar ações especificamente motoras dos seus alunos bem como se esse tipo de observação faz parte de seu planejamento pedagógico.

\section{MATERIAIS E MÉTODO}

A amostra foi constituída por trinta professores de sala, sendo vinte de duas escolas públicas e dez de duas escolas particulares. Todos trabalham com crianças entre oito e nove anos de idade que estão cursando, respectivamente, a segunda e a terceira séries do Ensino Fundamental. Também participaram deste estudo três professores de Educação Física, sendo dois das escolas particulares e um das escolas públicas.

Antes da aplicação da lista de checagem, esta foi apresentada a 15 professoras do curso de Pedagogia da UNIVÁS, na cidade de Cambuí-MG, para verificar como seria o entendimento das instruções da lista de checagem. A pós o levantamento da avaliação dessas professoras, ficou estabelecido que: a) seria colocada a alternativa Não Sei (NS) ao final das opções em cada item, o que não existe na lista de checagem original; b) o professor de sala de aula deveria responder os itens com caneta azul; caso ele não soubesse responder algum item, o professor de Educação Física deveria fazêlo com caneta vermelha e, se nenhum dos dois profissionais soubessem responder a algum dos itens, o responsável pela criança deveria 
fazê-lo com caneta preta; c) a numeração dos itens foi estabelecida de maneira contínua independentemente da seção - e não de 1 a 12 por seção como na lista de checagem original - para evitar possível confusão com a repetição da numeração dos itens. Antes de iniciar a pesquisa, foi feito contato com os supervisores e, em al guns casos, com o próprio diretor das escolas envolvidas, a fim de explicar os procedimentos e obter autorização para falar com os professores. A seguir, uma reunião foi realizada com os professores de sala de aula para esclarecer o objetivo e importância do trabal ho, pedir a participação voluntária de cada um, além de enfatizar a necessidade do compromisso e da seriedade ao responder cada item da lista de checagem.

Foi realizada outra reunião, dessa vez com os professores de Educação Física. Os mesmos procedimentos foram utilizados, porém deixando claro que eles complementariam as respostas dos professores de sala de aula.

Foi feito um pedido de autorização aos responsáveis pelas crianças, por escrito, com explicação a respeito dos objetivos da pesquisa.

Todos os professores realizaram a leitura das instruções e foram esclarecidos a respeito de cada passo a ser seguido. Ressaltou-se a necessidade de atenção, responsabilidade e certeza das informações a serem passadas mediante as respostas de cada seção. Cada item, das cinco seções da lista de checagem, foi lido e questionado.

Os professores de sala de aula ficaram responsáveis por observar três crianças de sua classe no momento, sendo uma menina (a primeira da lista de chamada), um menino (o primeiro da lista de chamada) e uma outra criança (menino ou menina) escoIhida pelo professor por reconhecer nela dificuldades motoras.

Os professores de sala de aula iniciaram seu trabaIho, tendo sido estabelecido que eles teriam no máximo quinze dias para responder a lista de checagem. A pós duas semanas, as listas de checagem foram passadas aos professores de Educação Física. Na reunião de pais, as questões que os professores (tanto o de Educação Física, quanto os de sala de aula) não souberam responder, foram respondidas pelo responsável da criança observada.

\section{RESULTADOS E DISCUSSÃO}

Para chegar às análises fundamentais desse estudo, foram estabelecidas as seguintes perguntas como elementos norteadores: 1) Os professores compreendem o que representam, na forma de comportamento motor, os itens descritos? 2) Eles dispõem de tempo para observar ações especificamente motoras? 3) Esse tipo de observação faz parte do seu planejamento pedagógico?

Sobre a questão 1, "Os professores compreendem o que representam, na forma de comportamento motor, os itens descritos?", a análise das respostas evidenciou que, para a seção 1, nos Itens 07 e 12 houve dúvidas pelo fato de duas tarefas estarem descritas no mesmo item; nos itens 06 e 11, não foi entendido qual era o grau de dificuldade da tarefa. Nos itens mencionados, pode-se observar que um fator intrínseco à lista tal como a dificuldade de compreensão sobre o que especificamente $o$ item pede que se observe e tarefas similares pedi das em um mesmo item, interferem na interpretação do professor. Na seção 2, nos itens 16 e 17, houve dificuldade na diferenciação entre as habilidades locomotoras saltito e galope. Um fator extrínseco, tal como formação profissional não compatível com a tarefa a ser observada, atrapalhou a interpretação do professor de sala. Ainda na seção 2, as respostas aos itens 19 e 20, dependem das características da instituição escolar, as quais também atuam como um fator extrínseco limitante na aplicabilidade da lista.

$\mathrm{Na}$ seção 3, os itens 26 e 36: dez professores de sala (escolas particulares) não responderam aos referidos itens, deixando para os professores de Educação Física.

Nesses itens, o que se percebe é que os professores da escola pública têm um contato maior com os alunos, uma vez que a parte diversificada do currículo da escola pública é menor, isto implica que os professores de sala ficam mais tempo com as crianças, proporcionando maior oportunidade de reconhecimento das habilidades motoras das crianças. Na seção 4, o item 37 foi respondido por todos os professores de sala; o item 38 ficou para ser respondido pelo responsável; os itens 42; 43; 44; 45; 46 e 48 foram respondidos pelos professores de Educação Física e os itens 39 e 47 foram respondidos pelos professores de Educação Física (das escolas particulares) e pelos vinte professores de sala das escolas públicas. 
Fica claro nesses itens que os professores de sala das escolas particulares têm dificuldade em perceber ações motoras que fazem parte dessa seção da lista de checagem, não só por falta de formação especifica, como também por fal ta de mai or tempo de convívio com o aluno. Por isso, se faz necessária a colaboração do professor de Educação Física para responder a lista de checagem nas escolas particulares. $\mathrm{O}$ item 38 nos remete a infra-estrutura escolar, que na maioria dos casos não possui um espaço específico de recreação para os alunos, mesmo na escola particular.

Na seção 5, todos os itens foram respondidos pelo professor de sala. Essa seção refere-se a problemas comportamentais relacionados com dificuldades em sala de aula, portanto os professores de sala são capazes de observar e ter certeza ao emitir um julgamento sobre o comportamento do al uno.

Na questão 2, "A rotina dos professores brasileiros possibilita a observação dos comportamentos descritos nos itens?", os resultados mostraram que em três das cinco seções - 1, 2 e 5 -, os professores de sala sentiram-se seguros em responder a maioria dos itens. Nas outras duas seções, - 3 e 4 - em que houve al guma incerteza por parte do professor de sala, o professor de Educação Física respondeu com competência. Portanto é possível dizer que a rotina de trabalho dos professores de sala com os alunos possibilita boa observação de seus comportamentos. A questão 3, "A observação de comportamentos motores faz parte dos pressupostos pedagógicos dos professores brasileiros?", foi proposta posteriormente, oriunda de conversas com os professores durante a realização deste estudo. A resposta a esta pergunta é "Não", pois tais conversas evidenciaram que do professor de sala não é cobrado esse tipo de observação. Se algum comportamento muito exacerbado é freqüentemente demonstrado, é possível que o professor de sala vá procurar alguém com competência para resolver o problema, mas ações motoras simples do dia-a-dia e "incompetências" leves passam quase despercebidas. Contudo, quando foi solicitado, eles recordaram fatos de sala de aula como, por exemplo, comportamentos diferenciados ou fugas inesperadas de algumas tarefas, mas justificaram que esse comportamento "meio desastrado" de al guns "...é fase, é normal, quando crescer passa". Em suma, 92,2\% dos itens foram respondidos pelo professor de sala, 6,8\% pelo professor de Educação Física e apenas $1 \%$ pelo responsável. Os resultados dos das seções 1, 2 e 5 mostraram que a maioria dos professores de sala responderam a todos os itens. $\mathrm{Na}$ seção 3, os itens 25; 27; 28; 29; 30; 31; 32; 33; 34; 35 foram respondidos por todos professores de sala. Os itens 26 e 36 foram respondidos por vinte professores da sala e pelos professores de Educação Física. $\mathrm{Na}$ seção 4, os itens 42; 43; 44; 45; 46 e 48 foram respondidos pelos professores de Educação Física, os itens 47 e 39 foram respondidos pelos professores de Educação Física e por vinte professores de sala; todos os professores de sala responderam o item 37 e o item 38 ficou para ser respondido pelo responsável. Neste sentido, mediante a análise das listas de checagem respondidas, pode-se perceber que ação do professor de sala de aula em conjunto com o professor de Educação Física, mostrou ser a solução mais ade quada para o preenchimento da lista de checagem.

\section{CONSIDERAÇÕES FINAIS}

Para verificar a aplicabilidade do teste $A B C$ do Movimento é importante analisar se os professores compreendem o significado de todos os itens da lista de checagem; se a rotina dos professores possibilita a observação dos comportamentos descritos nos itens; se a observação de comportamentos motores faz parte dos pressupostos pedagógicos da escola; se o ambiente na escola propicia as observações propostas, em relação a equipamentos, e se os comportamentos descritos nos itens fazem parte do cotidiano das crianças brasileiras. Tal análise pode levar a sugestões de possíveis adaptações da lista de checagem a diferentes contextos.

O presente estudo evidenciou que, apesar das limitações, a aplicabilidade da lista de checagem do Teste $A B C$ do Movimento (9) é viável. A investigação sobre ferramentas diagnósticas do TDC, como é o caso do presente trabal ho, juntamente com estudos acerca da sua natureza possibilitarão propostas de programas de intervenção sérios e eficientes.

\section{CORRESPONDÊNCIA}

\section{Jane A parecida de Oliveira Silva}

Rua Coronel Otávio Meyer, № 20, apto 901 CEP: 37.550-000 - Pouso Alegre, MG, Brasil janeolives@ hotmail.com 


\section{REFERÊNCIAS BIBLIOGRÁFICAS}

1. Associação Psiquiátrica Americana (1995). Critérios de diagnósticos do DSM -IV. Referência Rápida. Porto Alegre: Artes Médicas.

2. Barnett AL, Henderson SE (1992). Some Observations on the Figure Drawings of Clumsy Children. British Journal of Educacional Psychology 62: 341-355.

3. Dwyer C, Mckenzie BJ (1994). Impairment of visual memory in children who are clumsy. A dapted Physical Activity Quarterly (11):179-189.

4. EDITORIAL (1962). Clumsy Children. British M edical Journal 22: 1665-1666.

5. Ferreira LF (2004). Identificação de crianças com transtorno do desenvolvimento da coordenação: a lista de checagem do teste $M$ A BC em foco. Dissertação de Mestrado. Escola de Educação Física e Esportes. Universidade de São Paulo, São Paulo.

6. Geuze R, Kalverboer A (1994) Tapping a rhythm: A problem of timing for the children who are clumsy or dyslexic. A dapted Physical Activity Quarterly 11: 203-213.

7. Gubbay SS, Ellis E, Walton JN, Court DM (1965) Clumsy Children A Study of Apraxic and Agnosic defects in 21 children. Brain (88): 295-312..

8. Hall DM (1988). Clumsy children. British M edical Journal 296: 375-376.

9. Henderson SE, Sugden DA (1992). M ovement A ssessment Battery for Children: London: Psychological Corporation.

10. Henderson SE (1987). The assessement of "clumsy" children: old and new approaches. Journal of Psychology and Psychiatry, 28(4): 511-527.

11. Kalverboer AF, de Vries, Van Dellen T (1990). Social behavior in clumsy children as rated by parents and teachers. In: A.F. Kalverboer (Ed.) D evelopmental Biopsychology: Experimental and $\mathrm{O}$ bservational Studies in Children at Risk. Ann Arbor: University of Michigan Press.

12. Kephart NC (1986). 0 aluno de aprendizagem lenta. Porto Alegre: Artes Médicas.

13. Lord R, Hulme C (1987). Perceptual Judgements of Normal and Clumsy Children. Developmental M edicine and Child N eurology 29: 250-257.

14. Losse A, Henderson SE, Elliman D, Hall D, Knight E, Jongmans MJ (1991). Clumsiness in children: do the grow out of it? A 10-year follow-up study. D evelopmental M edicine and Child N eurology 33: 55-68.

15. Mandich AD, Polatajko HJ, Rodger S (2003). Rites of passage: Understanding participation of children with developmental coordination disorder. Human M ovement Science, 22(4-5): 583-595

16. O'Beirne C, Larkin D, et al. (1994). Coordination Problems and Anaerobic Performance in Children. A dapted Physical Activity Q uarterly 11: 141-149.

17. Oliveira MA (2003). Controle de força e torque isométrico em crianças come sem desordem coordenativa desenvolvimental. Tese de Doutorado. Escola Superior de Educação Física, Universidade Federal do Rio Grande do Sul, Porto Alegre, Brasil.
18. Piek, J. P.; Dworcan, M.; Barrett, N.C.; Coleman, R. (2000). Determinants of self-worth in children with and without developmental coordination disorder. International Journal of Disability, Development and Education 47 (3): 259272.

19. Schoemaker M, Kalverboer AF (1994). Social and affective problems of children who are clumsy: How early do they begin? A dapted Physical A ctivity Q uarterly 11: 130-140.

20. Schoemaker MM, Smits-Engelsman BC, Jongmans $M$ (2003). Psychometric properties of the Movement Assessment Battery for Children-Checklist as a screening instrument for children with a developmental co-ordination disorder. British Journal of Educational Psychology, 73 (Part3): 425-441.

21. Skinner RA, Piek JP (2001). Psychosocial implications of poor motor coordination in children and adolescents. H uman M ovement Science 20(1-2): 73-94.

22. Smits-Engelsman BCM, Henderson SE, Michels CGJ (1998). The Assessment of children with developmental coordination disorders in the Netherlands: the relationship between the movement assessment battery for children and the korperkoordinations test fur kinder. H uman M ovement Science 17: 699-709.

23. Smyth MM, Anderson HI (2000). Coping with clumsiness in the school playground: Social and physical play in children with coordination impairments. British Journal of Developmental Psychology 18: 389-413.

24. Smyth, M. M. \& Anderson, H. I. (2001). Football participation in the primary school playground: The role of coordination impairments. British Journal of D evelopmental Psychology 19: 369-379.

25. Souza CJF (2004). 0 teste ABC do M ovimento em crianças de ambientes diferentes. Dissertação de Mestrado. Escola de Educação Física e Esportes. Universidade de São Paulo, São Paulo.

26. Sugden DA, Wright HC (1998) M otor Coordination Disorders in Children. Sage publication: London.

27. Valentini NC (1997) The Influence of Two M otor Skill Interventions on the M otor Skill Performance, Perceived Physical Competence, and Intrinsic M otivation of Kindergarten Children. Thesis (Master of Science). Auburn, Alabama, Graduate Faculty of Auburn University, December 15.

28. Wall AE, Mcclements J, Bouffard M, Findlay $H$, Taylor MJA (1985). Knowledge-based approach to motor development: Implications for the physically awkward. A dapted Physical A ctivity Q uarterly 2: 21-42.

29. Wright H, Sugden DA, NG R, Tan J (1994). Identification of Children With Movement Problems in Singapore: Usefulness of the Movement ABC Checklist. A dapted Physical Activity Q uarterly 11:150-157.

30. Wright, H.C. (1997). Children with Developmental Coordination Disorder - A Review. European Journal Physical Education 2: 5-22. 